\title{
Residual thoracolumbar/lumbar curve is related to self-image after posterior spinal fusion for Lenke 1 and 2 curves in adolescent idiopathic scoliosis patients
}

\author{
Tetsuhiko Mimura, MD, ${ }^{1}$ Shota Ikegami, MD, PhD, ${ }^{1}$ Shugo Kuraishi, MD, PhD, ${ }^{1}$ \\ Masashi Uehara, MD, PhD, ${ }^{1}$ Hiroki Oba, MD, PhD, ${ }^{1}$ Takashi Takizawa, MD, PhD, ${ }^{1}$ \\ Ryo Munakata, MD, ${ }^{1}$ Terue Hatakenaka, MD, ${ }^{1}$ Michihiko Koseki, PhD, ${ }^{2}$ and \\ Jun Takahashi, MD, PhD'
}

${ }^{1}$ Department of Orthopaedic Surgery, Shinshu University School of Medicine, Matsumoto; and 'Faculty of Textile Science and Technology, Shinshu University, Ueda, Nagano, Japan

OBJECTIVE Although it is well known that major curve severity in adolescent idiopathic scoliosis (AIS) is inversely related to self-image, surgeons often encounter patients who complain of low self-image with preoperatively mild curves or postoperatively well-corrected main curves, suggesting the presence of other factors. This study examined factors contributing to self-image in AIS.

METHODS A total of 86 consecutive patients who underwent posterior spinal fusion for AIS Lenke 1 or 2 curves and were followed for a minimum of 2 years were included in this study of patient self-image based on data that included scores reported on the Scoliosis Research Society survey (SRS-22r). The authors evaluated sex, BMI, Risser grade, age, angle of trunk rotation, Cobb angle of the main thoracic (MT) curve, Cobb angle of the thoracolumbar/lumbar (TL/L) curve, apical vertebral translation (AVT), T5-12 kyphotic angle, and clavicular angle. Univariate and multivariate general linear models were employed to identify preoperative and 2-year postoperative factors that impact self-image.

RESULTS Univariate analysis revealed no significant correlation between preoperative MT curve Cobb angle and SRS$22 r$ self-image score $(p=0.51)$, although patients with a higher MT curve AVT had a significantly worse preoperative self-image $(p<0.01)$. Two years postoperatively, larger Cobb angle of the $T L / L$ curve $(p=0.01)$ and higher Risser grade $(p=0.03)$ resulted in significantly lower self-image scores. In multivariate testing, preoperative MT curve AVT remained significantly related to diminished self-image $(p<0.01)$. Two years later, higher $T L / L$ curve $(p<0.01)$, Risser grade $(p=$ $0.03)$, and MT curve AVT ( $p=0.03$ ) had significant associations with lower self-image scores.

CONCLUSIONS Preoperative MT curve AVT appears more strongly related to self-image than does Cobb angle. Two years postoperatively, persistent TL/L region curvature and high Risser grade may also be associated with diminished patient self-image.

https://thejns.org/doi/abs/10.3171/2020.2.PEDS19656

KEYWORDS adolescent idiopathic scoliosis; Scoliosis Research Society-22r; self-image; posterior spinal fusion; cosmesis; Cobb angle; spine

A DOLESCENT idiopathic scoliosis (AIS) is a pathology that affects multiple domains of health-related quality of life (QOL).,2 The Scoliosis Research Society survey (SRS-22r) is a commonly used patientreported outcome tool to assess health-related QOL for scoliosis patients. ${ }^{3}$

Cosmesis is a critical factor for patients with AIS. Thus, an important surgical goal is the improvement of physical appearance. Although it is well known that main curve severity in AIS is related to self-image, ${ }^{4-6}$ surgeons often encounter patients who complain of low self-image with preoperatively mild curves or postoperatively well-corrected main curves, suggesting the presence of additional factors that contribute to self-image. Other factors that influence cosmetic appearance and self-image in AIS include deformities such as a rib hump ${ }^{6,7}$ along with such patient char-

ABBREVIATIONS AIS = adolescent idiopathic scoliosis; $A T R=$ angle of trunk rotation; AVT = apical vertebral translation; MT = main thoracic; SRS-22 $r$ = Scoliosis Research Society survey; TL/L = thoracolumbar/lumbar.

SUBMITTED October 28, 2019. ACCEPTED February 21, 2020.

INCLUDE WHEN CITING Published online April 24, 2020; DOI: 10.3171/2020.2.PEDS19656. 
acteristics as age and psychological factors ${ }^{8-10}$ However, the relationship between curve severities other than that of the main thoracic (MT) curve, including thoracolumbar/ lumbar (TL/L) curve severity, and self-image is unknown. We hypothesized that the MT curve severity as well as the TL/L curve severity and other patient features could influence the self-image of AIS patients with a Lenke type 1 or 2 curve.

In this study we analyzed perioperative factors contributing to diminished self-image in AIS, including several radiographic parameters and patient characteristics.

\section{Methods}

A total of 93 consecutive AIS patients underwent posterior spinal fusion for an AIS Lenke 1 or 2 curve at our hospital between October 2005 and April 2017. Among them, 86 patients ( 77 females and 9 males) who were followed for a minimum of 2 years and had completed SRS$22 \mathrm{r}$ outcome measurements preoperatively and at 2 years postoperatively were retrospectively enrolled. All patients were treated with an all-pedicle screw construct. Mean \pm SD age was $15.1 \pm 2.4$ years (range $12-24$ years). Informed consent was obtained from all patients and guardians. This study was approved by the institutional ethics review board (no. 4113).

We evaluated the parameters of age, Risser grade, BMI, findings from total spine anteroposterior and lateral standing radiographs, MT curve, angle of trunk rotation (ATR), and pre- and postoperative SRS-22r self-image scores. ATR was measured with a scoliometer. Radiographic parameters included MT and TL/L curve Cobb angle, MT curve apical vertebral translation (AVT), T5-12 kyphotic angle, and clavicular angle (absolute value). Lenke type was $1 \mathrm{~A}$ in 38 patients, $1 \mathrm{~B}$ in 12 patients, $1 \mathrm{C}$ in 19 patients, $2 \mathrm{~A}$ in 13 patients, $2 \mathrm{~B}$ in 3 patients, and $2 \mathrm{C}$ in 1 patient. All radiographic measurements were performed by a trained orthopedic surgeon who was uninvolved in the surgeries.

Univariate and multivariate general linear models were employed to identify factors associated with self-image at 2 years after surgery for AIS. The main outcome of interest was 2-year postoperative SRS-22r self-image score. Candidate variables for the multivariate general linear model were selected via a univariate general linear model using the selection criterion of $p<0.2$. Comparisons of preoperative and 2-year postoperative SRS-22r scores were performed using paired t-tests.

Post hoc studies were carried out to test whether screw density or distal adding-on could have impacted postoperative self-image in association with residual TL/L curve. Distal adding-on was defined as 1) an increase in Cobb angle of $\geq 5^{\circ}$ and distalization of the end vertebra or 2) a change in disc angulation of $\geq 5^{\circ}$ below the lowest instrumented vertebra between the initial and 2-year follow-up radiographs. ${ }^{11}$ Screw density was expressed as the number of screws per fusion segment. To reveal the relationship between distal adding-on and postoperative self-image, comparisons of 2-year postoperative SRS-22r self-image scores in groups either with or without distal adding-on were performed using Welch's t-test. To investigate the relationship between screw density and 2-year postoper- ative SRS-22r self-image scores, univariate analysis was carried out using Pearson's correlation coefficient.

For all analyses, a p value of $<0.05$ was considered statistically significant. Statistical analyses were carried out using the statistical package R, version 3.2.0 (available at http://www.r-project.org).

\section{Results}

No severe complications due to surgical invasion, such as neurological deficit, pulmonary complication, infection, or fatal blood loss, were observed. No revision surgery due to complications was performed in this series.

The mean \pm SD SRS-22r self-image score was significantly improved at 2 years postoperatively $(3.9 \pm 0.7$ vs preoperative $2.7 \pm 0.5, \mathrm{p}<0.01$ ). Preoperative (Table 1) univariate analysis revealed no significant correlation between the preoperative MT curve Cobb angle and the preoperative SRS-22r self-image score $(\mathrm{p}=0.51)$, although a higher AVT value of the MT curve was significantly associated with a lower preoperative self-image $(\mathrm{p}<0.01)$. Higher MT curve AVT value $(\mathrm{p}<0.01)$ remained significant after multivariate testing.

At 2 years after surgery (Table 2), higher values for the Cobb angle value of the TL/L curve and the Risser grade were associated with significantly worse SRS-22 self-image scores in univariate analysis $(\mathrm{p}=0.01$ and $\mathrm{p}=0.03$, respectively) and in multivariate analysis $(\mathrm{p}<0.01$ and $\mathrm{p}=$ 0.03 , respectively, ). A higher AVT value of the MT curve also had a significant impact on worse self-image in multivariate testing $(\mathrm{p}=0.03)$. No remarkable relationships were observed for sex, BMI, age, MT curve ATR, T5-12 kyphotic angle, clavicular angle, or preoperative SRS-22r self-image score.

Three patients (3\%) experienced distal adding-on. SRS22r self-image scores did not differ significantly between patients with and without distal adding-on (4.6 \pm 0.4 vs 3.9 $\pm 0.7, p=0.07)$. The mean \pm SD screw density was $1.5 \pm$ 0.2 . The correlation coefficient between screw density and 2-year postoperative SRS-22r self-image scores was low at $-0.15(\mathrm{p}=0.17)$.

\section{Case Presentations}

The following 2 representative cases illustrate the relationship between residual TL/L curve and SRS-22r selfimage score.

\section{Case 1}

A 12-year-old girl presented with AIS (Fig. 1). Her MT curve from $\mathrm{T} 6$ to $\mathrm{L} 1$ was $59^{\circ}$ and her curve pattern was Lenke type $1 \mathrm{AN}$. The TL/L curve was $33^{\circ}$. The patient's preoperative SRS-22r self-image score was 1.8. She underwent posterior spinal fusion with skip pedicle screw fixation from T4 to L3. Postoperative radiographs obtained 2 years after surgery showed the MT and TL/L curves to be $14^{\circ}$ and $4^{\circ}$, respectively. The patient's 2 -year postoperative SRS-22r self-image score was good at 4.8 .

\section{Case 2}

A 14-year-old girl presented with AIS (Fig. 2). Her MT 
TABLE 1. Analysis results for the association of variables with the preoperative SRS-22r self-image score

\begin{tabular}{|c|c|c|c|c|}
\hline \multirow[b]{2}{*}{ Variable* $^{*}$} & \multicolumn{2}{|c|}{ Crude } & \multicolumn{2}{|c|}{ Adjusted } \\
\hline & Value & $p$ Value & Value & $\mathrm{p}$ Value \\
\hline Sex (male) & $0.11 \pm 0.18$ & 0.55 & & \\
\hline BMI $(+1$ kg/m²) & $0.01 \pm 0.03$ & 0.66 & & \\
\hline Risser grade (+1 grade) & $-0.03 \pm 0.04$ & 0.49 & & \\
\hline Age (+10 yrs) & $-0.08 \pm 0.23$ & 0.73 & & \\
\hline $\operatorname{MT} \operatorname{ATR}\left(+10^{\circ}\right)$ & $-0.16 \pm 0.12$ & 0.19 & $0.10 \pm 0.15$ & 0.49 \\
\hline \multicolumn{5}{|l|}{ Radiographic parameters } \\
\hline MT Cobb angle $\left(+10^{\circ}\right)$ & $-0.05 \pm 0.07$ & 0.51 & & \\
\hline TL/L Cobb angle $\left(+10^{\circ}\right)$ & $0.01 \pm 0.06$ & 0.82 & & \\
\hline MT AVT $(+1 \mathrm{~cm})$ & $-0.09 \pm 0.03$ & $<0.01$ & $-0.15 \pm 0.04$ & $<0.01$ \\
\hline T5-12 kyphotic angle $\left(+10^{\circ}\right)$ & $-0.06 \pm 0.05$ & 0.22 & & \\
\hline Clavicular angle absolute value $\left(+1^{\circ}\right)$ & $-0.04 \pm 0.04$ & 0.36 & & \\
\hline
\end{tabular}

Data are expressed as the value \pm SE unless otherwise indicated. Boldface type indicates statistical significance at $p<$ 0.05 . Adjusted values were estimated using a multivariate general linear model. Candidate variables were selected via a univariate general linear model using the selection criterion of $p<0.2$.

* Positive (+) values in parentheses indicate adjusted values estimated using a multivariate general linear model.

curve from T5 to T11 was $59^{\circ}$ and her curve pattern was Lenke type $1 \mathrm{C}-$. The TL/L curve was $42^{\circ}$. The patient's preoperative SRS-22r self-image score was 3.0. Posterior spinal fusion with skip pedicle screw fixation from T3 to T12 was performed. Postoperative radiographs 2 years postoperatively revealed MT and TL/L curves of $22^{\circ}$ and $25^{\circ}$, respectively. The patient's 2-year postoperative SRS$22 \mathrm{r}$ self-image score was poor at 3.2.

\section{Discussion}

This study demonstrated that the mean SRS-22r self- image score increased significantly from 2.7 to 3.9 in AIS patients who underwent posterior spinal fusion. This improvement was considered to have clinical importance based on Carreon et al.'s reported minimum clinically important difference for the self-image domain of $0.98 . .^{12}$

Adolescents with scoliosis are more likely to be dissatisfied with their appearance and to fear that their bodies are developing abnormally than are adolescents without scoliosis..$^{13}$ One of the most obvious differences from a healthy person is a spinal deformity. Several reports have attempted to identify the correlations between deformity measures and self-image. Wang et al. found that self-

TABLE 2. Analysis results for the association of variables with the 2-year postoperative SRS-22r self-image score

\begin{tabular}{|c|c|c|c|c|}
\hline \multirow[b]{2}{*}{ Variable* } & \multicolumn{2}{|c|}{ Crude } & \multicolumn{2}{|c|}{ Adjusted } \\
\hline & Value & $p$ Value & Value & $\mathrm{p}$ Value \\
\hline Sex (male) & $-0.06 \pm 0.25$ & 0.80 & & \\
\hline $\mathrm{BMI}\left(+1 \mathrm{~kg} / \mathrm{m}^{2}\right)$ & $-0.04 \pm 0.04$ & 0.34 & & \\
\hline Risser grade (+1 grade) & $-0.11 \pm 0.05$ & 0.03 & $-0.11 \pm 0.05$ & 0.03 \\
\hline Age ( +10 years) & $-0.09 \pm 0.32$ & 0.77 & & \\
\hline MT ATR 2 years postop $\left(+10^{\circ}\right)$ & $-0.24 \pm 0.21$ & 0.25 & & \\
\hline \multicolumn{5}{|l|}{ Radiographic parameters 2 years postop } \\
\hline MT Cobb angle $\left(+10^{\circ}\right)$ & $-0.14 \pm 0.10$ & 0.19 & $-0.03 \pm 0.13$ & 0.82 \\
\hline TL/L Cobb angle $\left(+10^{\circ}\right)$ & $-0.26 \pm 0.10$ & 0.01 & $-0.39 \pm 0.14$ & $<0.01$ \\
\hline MT AVT (+1 cm) & $-0.12 \pm 0.09$ & 0.17 & $-0.20 \pm 0.09$ & 0.03 \\
\hline T5-12 kyphotic angle $\left(+10^{\circ}\right)$ & $-0.03 \pm 0.09$ & 0.74 & & \\
\hline Clavicular angle absolute value $\left(+1^{\circ}\right)$ & $-0.10 \pm 0.06$ & 0.09 & $-0.10 \pm 0.05$ & 0.05 \\
\hline Preoperative SRS-22r self-image score & $0.11 \pm 0.15$ & 0.48 & & \\
\hline
\end{tabular}

Data are expressed as the value \pm SE unless otherwise indicated. Boldface type indicates statistical significance at $p<$ 0.05 . Adjusted values were estimated using a multivariate general linear model. Candidate variables were selected via a univariate general linear model using the selection criterion of $p<0.2$.

* Positive (+) values in parentheses indicate adjusted values estimated using a multivariate general linear model. 


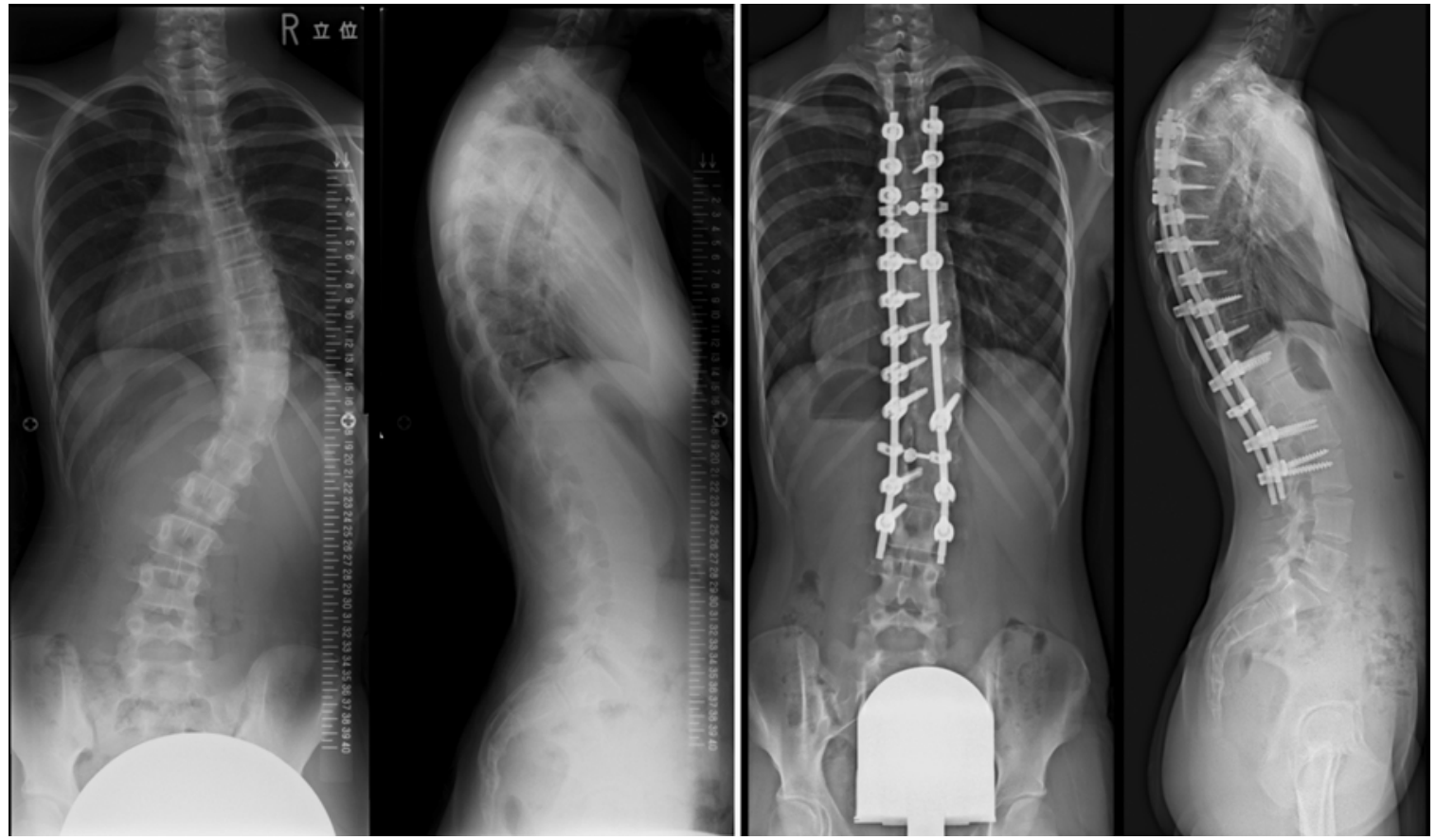

FIG. 1. Case 1. Preoperative (left) and 2-year postoperative (right) radiographs of Lenke type $1 A N$ thoracic AIS. The TL/L curve was $4^{\circ}$ postoperatively. The 2-year postoperative SRS-22r self-image score was good at 4.8 .

image correlated negatively with MT curve Cobb angle and AVT in AIS patients who first visited an orthopedic center. ${ }^{6}$ Asher et al. investigated AIS patients who underwent surgery posteriorly and found that the Cobb angle of the MT curve was associated with the SRS-22 self-im- age domain score, while trunk deformity magnitude was not. ${ }^{4}$ Theologis et al. reported that Cobb angle and lateral asymmetry measured on radiographs showed poor correlations with cosmetic spinal scores from ratings of clinical photographs by 10 nonmedical judges. ${ }^{7}$ Wilson et al. ana-
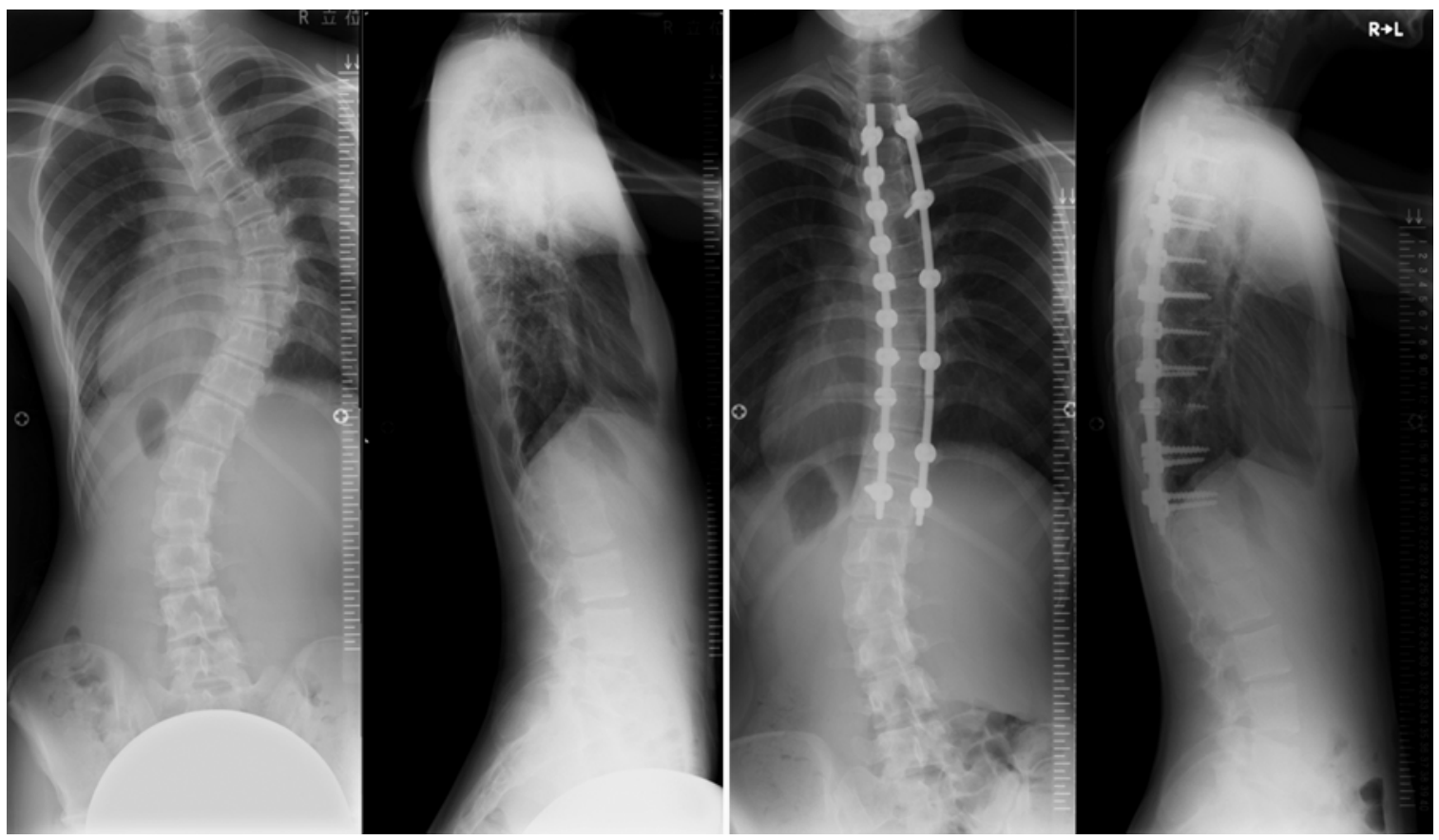

FIG. 2. Case 2. Preoperative (left) and 2-year postoperative (right) radiographs of Lenke type $1 C$ - thoracic AIS. The TL/L curve was $25^{\circ}$ postoperatively. The 2-year postoperative SRS-22r self-image score was poor at 3.2. 
lyzed AIS patients with nonoperative, preoperative, and surgically treated curves to reveal that the Cobb angle of the major deformity had a negative association with selfimage score..$^{14}$ In our study, the MT curve Cobb angle did not correlate remarkably with self-image, whereas the MT curve AVT was significantly associated with self-image both before surgery and at 2 years afterwards. Based on these findings, surgical reduction of AVT may provide significant improvements in postoperative self-image score.

Previous studies have demonstrated that a rib protuberance also influences cosmetic appearance and self-image in AIS. ${ }^{6,7}$ However, MT curve ATR was not observed to significantly impact self-image in our cohort. Most analyses to date have evaluated the relationship between selfimage and radiographic parameters of the major curve, with few focusing on residual lumbar curvature. Larson et al. reported that patients with long fusion lengths might be more pleased with the appearance of their back since a selective-fusion group had lower self-image scores than did a long-fusion group after surgery. ${ }^{15}$ The authors considered this result to be due to patient displeasure with residual lumbar curvature. In support of this finding, our study revealed the presence of a residual TL/L curve to be significantly related to self-image after surgery. Waistline perception may impact self-image, even if the main curve is well corrected by surgery. Accordingly, extending spinal fusion distally may be effective to reduce a residual lumbar curve, such as for spontaneous lumbar curve correction of a Lenke type 1 curve. ${ }^{16}$ However, because it is advisable to preserve as many caudal motion segments as possible to decrease the risk of disc degeneration ${ }^{17}$ and enable a return to athletic activity, ${ }^{18,19}$ distal fusion extension for better self-image is not a pragmatic option. Instead, patients should be informed of the possibility of lumbar curvature after surgery to help them accept any residual deformity.

Scoliosis is a deformity that appears in late childhood and adolescence. As Nathan argues, older scoliosis patients possess greater sensitivity to physical attractiveness and more acute awareness of their deformities, which may have psychological consequences. ${ }^{8}$ Other reported studies have also shown that psychological factors affect the self-image of adolescent scoliosis patients. ${ }^{9,10}$ In our study, a higher Risser grade was significantly related to worse self-image scores at 2 years after surgery in univariate and multivariate analyses. According to our univariate analysis, diminished flexibility may have influenced the residual lumbar curve, which is related to self-image. In multivariate analysis with adjustment for radiographic parameters, the significance of higher Risser grade likely reflected the psychological aspect of age in relation to self-image.

\section{Study Limitations}

This study had several limitations. First, it was performed in a patient group of limited size and had a retrospective design. Second, self-image in AIS was measured by SRS-22r self-image score alone, a method that might not have included all aspects of image assessment. For example, Bastrom et al. reported that the SRS-22 demonstrated an extensive ceiling effect at 2 years postoperatively and was psychometrically inferior in a postopera- tive population. ${ }^{20}$ The inclusion of other scales is therefore needed in future investigations. Third, since only patients who underwent posterior spinal fusion were included in this study, the effect of nonoperative mild deformity on self-image remains unknown. Fourth, as this series included Japanese patients only, there may have been factors impacting self-image that differed from those in other populations.

\section{Conclusions}

In summary, posterior spinal fusion for AIS significantly improved SRS-22r self-image scores. Perioperative AVT of the MT curve appears to be more strongly related to self-image than does Cobb angle. Two years after surgery, persistent curvature of the TL/L region and higher Risser grade may decrease gains in self-image. Informing patients preoperatively regarding the risk of residual lumbar curvature may help them to better accept possible postoperative deformity.

\section{References}

1. Asher MA, Min Lai S, Burton DC. Further development and validation of the Scoliosis Research Society (SRS) outcomes instrument. Spine (Phila Pa 1976). 2000;25(18):2381-2386.

2. Haher TR, Gorup JM, Shin TM, et al. Results of the Scoliosis Research Society instrument for evaluation of surgical outcome in adolescent idiopathic scoliosis. A multicenter study of 244 patients. Spine (Phila Pa 1976). 1999;24(14):1435-1440.

3. Asher M, Min Lai S, Burton D, Manna B. The reliability and concurrent validity of the scoliosis research society-22 patient questionnaire for idiopathic scoliosis. Spine (Phila Pa 1976). 2003;28(1):63-69.

4. Asher M, Lai SM, Burton D, Manna B. Spine deformity correlates better than trunk deformity with idiopathic scoliosis patients' quality of life questionnaire responses. Stud Health Technol Inform. 2002;91:462-464.

5. Parent EC, Hill D, Mahood J, et al. Discriminative and predictive validity of the scoliosis research society-22 questionnaire in management and curve-severity subgroups of adolescents with idiopathic scoliosis. Spine (Phila Pa 1976). 2009;34(22):2450-2457.

6. Wang L, Wang YP, Yu B, et al. Relation between self-image score of SRS-22 with deformity measures in female adolescent idiopathic scoliosis patients. Orthop Traumatol Surg Res. 2014;100(7):797-801.

7. Theologis TN, Jefferson RJ, Simpson AH, et al. Quantifying the cosmetic defect of adolescent idiopathic scoliosis. Spine (Phila Pa 1976). 1993;18(7):909-912.

8. Nathan SW. Coping with disability and the surgical experience: body image of scoliotic female adolescents. Clin Pediatr (Phila). 1978;17(5):434-440.

9. Noonan KJ, Dolan LA, Jacobson WC, Weinstein SL. Longterm psychosocial characteristics of patients treated for idiopathic scoliosis. J Pediatr Orthop. 1997;17(6):712-717.

10. Reichel D, Schanz J. Developmental psychological aspects of scoliosis treatment. Pediatr Rehabil. 2003;6(3-4):221-225.

11. Cho RH, Yaszay B, Bartley CE, et al. Which Lenke 1A curves are at the greatest risk for adding-on... and why? Spine (Phila Pa 1976). 2012;37(16):1384-1390.

12. Carreon LY, Sanders JO, Diab M, et al. The minimum clinically important difference in Scoliosis Research Society-22 Appearance, Activity, And Pain domains after surgical correction of adolescent idiopathic scoliosis. Spine (Phila Pa 1976). 2010;35(23):2079-2083. 
13. Payne WK III, Ogilvie JW, Resnick MD, et al. Does scoliosis have a psychological impact and does gender make a difference? Spine (Phila Pa 1976). 1997;22(12):1380-1384.

14. Wilson PL, Newton PO, Wenger DR, et al. A multicenter study analyzing the relationship of a standardized radiographic scoring system of adolescent idiopathic scoliosis and the Scoliosis Research Society outcomes instrument. Spine (Phila Pa 1976). 2002;27(18):2036-2040.

15. Larson AN, Fletcher ND, Daniel C, Richards BS. Lumbar curve is stable after selective thoracic fusion for adolescent idiopathic scoliosis: a 20-year follow-up. Spine (Phila Pa 1976). 2012;37(10):833-839.

16. Takahashi J, Newton PO, Ugrinow VL, Bastrom TP. Selective thoracic fusion in adolescent idiopathic scoliosis: factors influencing the selection of the optimal lowest instrumented vertebra. Spine (Phila Pa 1976). 2011;36(14):1131-1141.

17. Lonner BS, Ren Y, Upasani VV, et al. Disc degeneration in unfused caudal motion segments ten years following surgery for adolescent idiopathic scoliosis. Spine Deform. 2018;6(6):684-690.

18. Fabricant PD, Admoni S, Green DW, et al. Return to athletic activity after posterior spinal fusion for adolescent idiopathic scoliosis: analysis of independent predictors. J Pediatr Orthop. 2012;32(3):259-265.

19. Lehman RA Jr, Kang DG, Lenke LG, et al. Return to sports after surgery to correct adolescent idiopathic scoliosis: a survey of the Spinal Deformity Study Group. Spine $J$. 2015;15(5):951-958.

20. Bastrom TP, Bartley C, Marks MC, et al. Postoperative perfection: ceiling effects and lack of discrimination with both SRS-22 and -24 outcomes instruments in patients with adolescent idiopathic scoliosis. Spine (Phila Pa 1976). 2015;40(24):E1323-E1329.

\section{Disclosures}

The authors report no conflict of interest concerning the materials or methods used in this study or the findings specified in this paper.

\section{Author Contributions}

Conception and design: Takahashi, Mimura. Acquisition of data: Mimura. Analysis and interpretation of data: Mimura. Drafting the article: Mimura. Critically revising the article: Takahashi. Reviewed submitted version of manuscript: all authors. Approved the final version of the manuscript on behalf of all authors: Takahashi. Statistical analysis: Mimura, Ikegami. Administrative/technical/material support: Kuraishi.

\section{Supplemental Information}

\section{Previous Presentations}

The abstract of this study was presented as an e-poster on July 17-20, 2019, at the 26th International Meeting on Advanced Spine Techniques, Amsterdam, the Netherlands.

\section{Correspondence}

Jun Takahashi: Shinshu University School of Medicine, Matsumoto, Nagano, Japan. jtaka@shinshu-u.ac.jp. 